\title{
Rooted tree graphs and the Butcher group: Combinatorics of elementary perturbation theory
}

\author{
William G. Faris \\ NYU Shanghai and University of Arizona
}

January 26, 2021

\begin{abstract}
The perturbation expansion of the solution of a fixed point equation or of an ordinary differential equation may be expressed as a power series in the perturbation parameter. The terms in this series are indexed by rooted trees and depend on a parameter in the equation in a way determined by the structure of the tree. Power series of this form may be considered more generally; there are two interesting and useful group structures on these series, corresponding to operations of composition and substitution. The composition operation defines the Butcher group, an infinite dimensional group that was first introduced in the context of numerical analysis. This survey discusses various ways of realizing these rooted trees: as labeled rooted trees, or increasing labeled rooted trees, or unlabeled rooted trees. It is argued that the simplest framework is to use labeled rooted trees.
\end{abstract}

\section{Introduction}

This year we celebrate the scientific contributions of Charles Newman. Chuck has worked in almost every aspect of mathematical physics. He can identify a significant problem, locate the appropriate framework, and find an unexpected path to a comprehensible solution. His insights and his generosity in sharing them are extraordinarily valuable to the community. Chuck has been my friend and colleague from years together at the University of Arizona and now at NYU Shanghai. It is a pleasure to dedicate this paper to such a distinguished scientist.

The paper is a largely expository survey of rooted trees and the Butcher group. The Butcher group is an infinite dimensional group associated with rooted trees. See $[8$ for the current status of this subject. Most expositions are in the framework of unlabeled rooted trees. The main message of the present work is that the combinatorics is simpler when formulated in terms of labeled rooted trees. In particular, group structures associated with rooted trees arise naturally from calculations using elementary calculus formulas. This is in the spirit of the theory of combinatorial species [1]. This same point of view is 
fruitful in the study of graph expansions in statistical mechanics [10] and of diagram expansions in quantum field theory [11].

The subject matter of the present work has two independent origins. One begins in 1963 with work of Butcher in numerical analysis. He discovered that a large class of numerical methods for ordinary differential equations may be expressed as sums indexed by rooted trees. Furthermore, these sums may be combined in a way that defines a group structure. This subject has become part of the lore of numerical analysis [6, 13, 14. It remains active; for instance see [16, 4] and works cited therein.

The other origin is research on renormalization in quantum field theory, starting with the 1998 contribution of Connes and Kreimer [9]. This work is usually presented in the language of combinatorial Hopf algebras. Many authors, for instance [5, 12, have investigated the relation between the Butcher group and problems in quantum field theory. Recently there has been an explosion of mathematics papers treating Hopf algebras associated with rooted trees. See for instance [7] and papers cited there.

The approach here begins by distinguishing basic rooted tree constructions. The starting point is a finite set $U$ known as the label set or vertex set. A labeled rooted tree on $U$ is a tree graph with vertex set $U$ together with a distinguished point $r$ in $U$. An increasing rooted tree is one for which the label set $U$ is linearly ordered and the labels increase with distance from the root $r$. An unlabeled rooted tree is an isomorphism class of labeled rooted trees.

- $\mathcal{A}[U]$ is all rooted trees on label set $U$.

- $\mathcal{A}^{\uparrow}[U]$ is all increasing rooted trees on linearly ordered label set $U$.

- $\tilde{\mathcal{A}}[n]$ is all unlabeled rooted trees with $n$ vertices.

The $\mathcal{A}$ notation is from the French "arbre". The relation between these constructions is that if $U$ has $n$ elements, then

$$
\mathcal{A}^{\uparrow}[U] \rightarrow \mathcal{A}[U] \rightarrow \tilde{\mathcal{A}}[n] .
$$

The first map is an injection, and the second map is a surjection.

The topics discussed include:

- Unlabeled, labeled, and increasing rooted trees

- Fixed point equations and ordinary differential equations

- The composition operation (Butcher group)

- The substitution operation

An appendix reviews calculus formulas as used in combinatorics.

Example: Figure 1 illustrates the distinction between labeled rooted trees and unlabeled rooted trees in the case of a vertex set with three elements. There are 9 labeled rooted trees. However there are only 2 unlabeled rooted trees. One of these has a symmetry that exchanges the two non-root vertices. II 


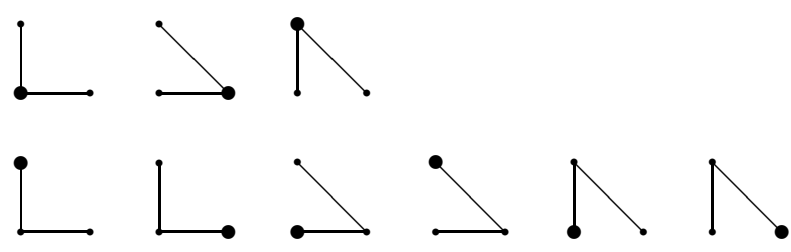

Figure 1: Labeled rooted trees on three vertices

\section{Labeled rooted trees}

Labeled rooted trees as functions Let $U$ be a non-empty finite set. Let $f: U \rightarrow U$ be a function with a single fixed point $r$ such that $U \backslash\{r\}$ has no non-empty invariant subset. Then $f$ defines a labeled rooted tree. In the following it will be more convenient to consider the function $f$ restricted to $U \backslash\{r\}$. This leads to the official definition of labeled rooted tree used here.

Let $U$ be a non-empty finite set. Let $r$ be a point in $U$, and let $T: U \backslash\{r\} \rightarrow U$ be a function that has no non-empty invariant subset. Then $T$ is called a labeled rooted tree. The set $U$ is the label set, and the point $r$ is the root. A point in $U$ is called a label or a vertex. Each ordered pair $(i, T(i))$ with $i \neq r$ is called an edge. The point $T(i)$ is called the predecessor of $i$.

The set of labeled rooted trees with label set $U$ is $\mathcal{A}[U]$. If $U$ is empty, then there are no labeled rooted trees on $U$, so $\mathcal{A}[\emptyset]=\emptyset$. If $T$ is in $\mathcal{A}[U]$, then $[T]=U$ is the label set of $T$. The number of points in the label set is $|T|=|U|$. The set of immediate successor points that map to $j$ in $U$ is $T^{-1}(j)$. The degree of $j$ is $\left|T^{-1}(j)\right|$, the number of immediate successor points. (The definition of degree used here is special to rooted trees; it is not the usual definition from graph theory.) A leaf is a vertex with degree zero. For a one-vertex tree $\bullet$ the root is a leaf.

If $b: W \rightarrow U$ is a bijection, then the map $T \mapsto T \circ b$ maps $\mathcal{A}[U]$ to $\mathcal{A}[W]$. Such a map is called a relabeling. Most interesting properties of labeled rooted trees are not affected by relabeling. It might seem reasonable to use a standard label set $U_{n}$ for each $n$. An obvious candidate is $[1, n]$, that is, the set $\{1, \ldots, n\}$. On the other hand, it is common to consider a labeled rooted tree on a subset of $U$, and that means that other labels sets are going to arise naturally.

The set $\mathcal{A}$ of all labeled rooted trees may be defined by choosing a label set $U$ with $|U|=n$ for each $n=1,2,3, \ldots$. For some purposes it is useful to adjoin an empty set object associated with the empty label set. The set of all labeled rooted trees with this extra object is written $\mathcal{A}_{\emptyset}$.

Labeled rooted trees as partially ordered sets A labeled rooted tree $T$ on a finite label set $U \neq \emptyset$ may be viewed as a partial order $\leq_{\text {tree }}$ on $U$. This is the unique partial order with the property that $T(j) \leq_{\text {tree }} j$ for every vertex $j \neq r$.

For each vertex $i$ there is a rooted tree $T_{i}$ whose vertex set consists of all vertices that are sent to $i$ by some iterate of $T$. The tree $T_{i}$ is the restriction of $T$ to this set. To say that $i \leq_{\text {tree }} j$ is the same as saying that $j$ is in the vertex set of the tree $T_{i}$.

The special feature of this partial order is that for each $j$ in $U$ the set of all $i \leq_{\text {tree }} j$ is linearly ordered with respect to the restriction of $\leq_{\text {tree }}$ to this 


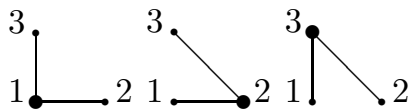

$11 \quad 22 \quad 33$

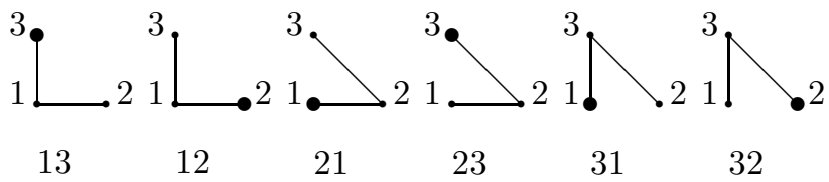

Figure 2: Labeled rooted trees on three vertices: Prüfer sequences

set. Furthermore, there is a least element in $U$, the root $r$. There can be one or more maximal elements; these are the leaves.

Labeled rooted trees as graphs A labeled tree $T$ on a non-empty finite set $U$ is a simple graph with $U$ as vertex set that is connected and has no cycles. For every pair of vertices $i \neq j$ there is a unique simple path connecting the two points. A labeled rooted tree $T$ on $U$ is equivalent to a labeled tree and a choice of root point in $U$. For each vertex $i$ other than the root, there is a unique edge from $i$ in the direction of the root and corresponding vertex $T(i)$. The usual way of picturing a labeled rooted tree is as a set together with tree graph and distinguished point.

Forests of labeled rooted trees Let $V$ be a finite set. Let $f: V \rightarrow V$ be a function with a set of fixed points $R$ such that $U \backslash R$ has no non-empty invariant subset. Then consider the function $f$ restricted to $U \backslash R$. This motivates the official definition of forest of labeled rooted trees.

A forest of labeled rooted trees on a set $V$ is a subset $R \subseteq V$ and a function $F: V \backslash R \rightarrow V$ such that $V \backslash R$ has no non-empty invariant subset. It is possible that $V=\emptyset$, in which case $R=\emptyset$, and $F$ is the empty function.

The most important fact about a forest of labeled rooted trees is that there is a set partition $\Gamma$ of $V$ with the following property. For each block $B$ of $\Gamma$ there is a unique $r$ in $R$ such that the restriction $F_{B}$ of $F$ to $B \backslash\{r\}$ is a labeled rooted tree. When $V=\emptyset$ this is the empty set partition.

Labeled rooted trees defined recursively A labeled rooted tree $T$ on $U$ has a recursive definition as a point $r$ in $U$ together with a forest of labeled rooted trees on $U \backslash\{r\}$. This forest $F$ is the restriction of $T$ to points in $U \backslash\{r\}$ that are not in $T^{-1}(r)$. The set of roots of the forest is $R=T^{-1}(r)$. The recursive definition ends when the tree consists only of a root; the forest is then empty.

Notation for labeled rooted trees A labeled rooted tree on a one-point set may be designated by its label $j$. A labeled rooted tree on a set with two or more points may be denoted by $j[$ ], where the forest of immediate successor rooted trees is listed (in some arbitrary order) inside the bracket. As an example, 


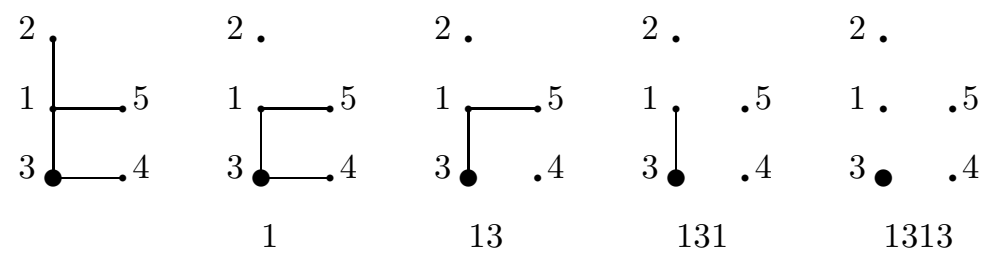

Figure 3: From labeled rooted tree to Prüfer sequence

consider the tree with root $c$ and with vertices $b, e$ that are sent to $c$ and vertices $a, d$ that are sent to $e$. In this notation the tree would be $c[b e[a d]]$.

Labeled rooted trees as sequences of vertices Consider a non-empty vertex set $U$ with $n$ elements. For the construction to follow it is necessary to impose a linear order on $U$. The result says that labeled rooted trees on $U$ correspond to sequences of $n-1$ elements.

Example: For $n=3$ the vertices of a labeled rooted tree may be numbered $1,2,3$. As shown below, each labeled rooted tree may be coded by a sequence of two numbers. For example, the tree 2[13] is coded by 22 , wile the tree $2[1[3]]$ is coded by 12 . There are nine sequences: three of them 11, 22, 33 correspond to one unlableled rooted tree, while six of them 12, 13, 21, 23, 31, 33 correspond to the other unlabeled rooted tree. See Figure 2 for the picture. II

Proposition 1 (Prüfer correspondence) Given non-empty label set $U$ with a given linear order, there is a bijection between the set of sequences $s$ of length $n-1$ of elements of $U$ and the set of labeled rooted trees $T$ in $\mathcal{A}[U]$. For each $j$ in $U$ the number of times the sequence $s$ assumes the value $j$ is the degree $\left|T^{-1}(j)\right|$.

Proof: It is easiest to see how to go from the labeled rooted tree $T$ to the corresponding sequence $s$. At each stage remove the smallest leaf and its corresponding edge from the tree. The value of $s$ at this stage is the vertex at the other end of this edge. When this is repeated $k-1$ times the final sequence value is the root. This procedure is illustrated in Figure 3.

Here is the construction to go from the sequence $s$ to the labeled rooted tree. The edges are restored in the same order as they were removed. At each stage add a new edge as follows. Take the smallest vertex that has not yet been used and that does not occur in the part of the sequence that has not been used. The edge then goes from this vertex to the next element of the sequence. See Figure 4 for a picture. $\|$

Proposition 2 (Cayley) The number of labeled rooted trees $T$ with $|T|=n$ vertices is $n^{n-1}$.

Proof: This famous result of Cayley results from the Prüfer correspondence. There are $n^{n-1}$ sequences of length $n-1$ in a set $U$ with $n$ elements. ॥ 


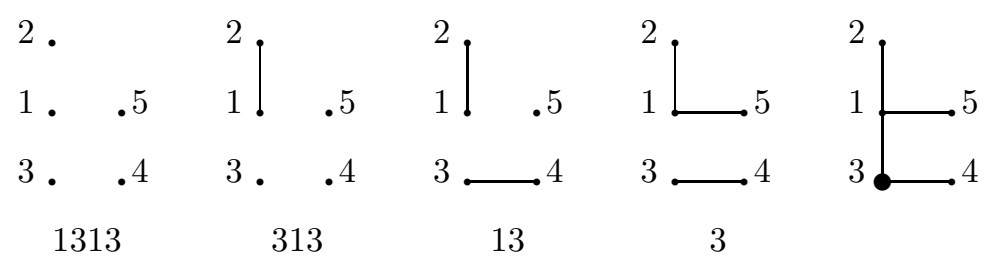

Figure 4: From Prüfer sequence to labeled rooted tree

\section{Unlabeled rooted trees}

The difficulty with unlabeled rooted trees is the general difficulty with unlabeled combinatorial structures (isomorphism classes of structures). This is the presence of symmetry. This is a well-studied topic; there are nice accounts in 1 and in 15 .

Unlabeled rooted trees via orbits of labeled rooted trees An unlabeled rooted tree is an isomorphism invariant of labeled rooted trees. It is convenient to fix the label set $U$. For each labeled rooted tree $T$ on $U$ there is a corresponding unlabeled rooted tree $\tau$. Here are the details.

Let $U$ be a non-empty set. Let $\mathcal{A}[U]$ be the set of labeled rooted trees on vertex set $U$. Each such tree is a function $T: U \backslash\{r\}$ to $U$. For each $T$ in $\mathcal{A}[U]$ and each bijection $b: U \rightarrow U$, the composite function $T \circ b$ is another labeled rooted tree in $\mathcal{A}[U]$. It is a function $T^{\prime}=T \circ b: U^{\prime} \backslash\left\{r^{\prime}\right\}$ to $U$, where $b r^{\prime}=r$. Two such labeled rooted trees $T, T^{\prime}$ are said to be isomorphic.

An unlabeled rooted tree $\tau$ with $n$ vertices is an object that corresponds to an isomorphism class of labeled rooted trees, where the label set has $n$ elements. The set of unlabeled rooted trees with $n$ vertices is denoted $\tilde{\mathcal{A}}[n]$. The set of all unlabeled rooted trees is denoted $\tilde{\mathcal{A}}$. There is no unlabeled rooted tree with zero vertices, but sometimes it is convenient to introduce an extra empty set object associated with zero vertices. The augmented set is written $\tilde{\mathcal{A}}_{\emptyset}$.

An unlabeled rooted tree $\tau$ has no underlying set and thus no vertices and no edges. Nevertheless, it may be pictured by any $T$ in the isomorphism class. There are various invariants of an unlabeled rooted tree $\tau$. Among them are the number of vertices $|\tau|$ and the number of vertices $v_{k}(\tau)$ of degree $k$. These are related by

$$
\sum_{k} k v_{k}(\tau)=|\tau|-1
$$

There are relatively few unlabeled rooted trees. The simplest (but nevertheless important) ones have 1 root and $n-1$ leaves. In the following such a tree will be denoted $\tau=n-1$. For this tree $|\tau|=n$ and $v_{0}(\tau)=n-1$, $v_{n-1}(\tau)=1$. Another simple class of unlabeled rooted trees are the linear trees with $v_{0}(\tau)=1$ and $v_{1}(\tau)=n-1$.

Group theory illuminates the situation. Fix a label set $U$ with $n$ elements. Let $\mathcal{G}$ be the permutation group of this set. This consists of all bijections $b: U \rightarrow U$. This group has $|\mathcal{G}|=n$ ! elements. The group $\mathcal{G}$ also acts on the set $\mathcal{A}[U]$ of labeled rooted trees with $n^{n-1}$ elements. For each bijection $b$, the map 


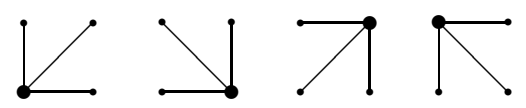

Figure 5: Orbit of a labeled rooted tree on four vertices: 4 trees, $\sigma(\tau)=6$

$T \rightarrow T \circ b$ is a map from $\mathcal{A}[U]$ to itself. The set $\tilde{\mathcal{A}}[n]$ of unlabeled rooted trees corresponds to the set of orbits under this action:

$$
\tilde{\mathcal{A}}[n] \cong \mathcal{A}[U] / \mathcal{G} .
$$

For each labeled rooted tree $T$, the corresponding unlabeled rooted tree $\tau$ is an abstract object corresponding to the orbit $\mathcal{G} T$ of $T$. The map from labeled rooted trees to unlabeled rooted trees may be summarized by the surjection

$$
\mathcal{A}[U] \rightarrow \tilde{\mathcal{A}}[n] .
$$

According to the theory of group actions, the size of the orbit $\mathcal{G} T$ of $T$ is the order $|\mathcal{G}|=n$ ! divided by the order $\left|\mathcal{G}_{T}\right|$, where $\mathcal{G}_{T}$ is the stabilizer subgroup of $T$. Thus

$$
|\mathcal{G} T|=\frac{n !}{\left|\mathcal{G}_{T}\right|} .
$$

The order $\left|\mathcal{G}_{T}\right|$ is the same for all $T$ in an orbit and hence may be denoted $\sigma(\tau)$, where $\tau$ is the unlabeled rooted tree corresponding to $T$. The number $\sigma(\tau)$ is the symmetry factor of $\tau$. The size of the orbit also depends only on $\tau$; it will be denoted $r(\tau)$. This gives the following basic result:

Proposition 3 For rooted trees with $|\tau|=n$ vertices the number of labeled rooted trees in $\mathcal{A}[U]$ per unlabeled rooted tree in $\tilde{\mathcal{A}}[n]$ is

$$
r(\tau)=\frac{n !}{\sigma(\tau)} .
$$

Example: There are labeled rooted trees on $n=4$ vertices that consist of a root and three leaves. The orbit $\mathcal{G} T$ of such a rooted tree is shown in Figure 5. For each rooted tree $T$ in this orbit, the corresponding stabilizer subgroup $\mathcal{G}_{T}$ has 6 elements, corresponding to the 6 permutations of the leaves. The symmetry factor is $\sigma(\tau)=6$. The number of labeled rooted trees in the orbit is $r(\tau)=24 / 6=4$. $\|$

There is an identity that expresses the fact that the sum over unlabeled rooted trees of the corresponding number of labeled rooted trees is the total number of labeled rooted trees.

Proposition 4 For unlabeled rooted trees $\tau$ with $|\tau|=n$ vertices the sum of the corresponding numbers $r(\tau)$ of labeled rooted trees in $\mathcal{A}[U]$ with $|U|=n$ is

$$
\sum_{\tau \in \tilde{\mathcal{A}}[n]} \frac{n !}{\sigma(\tau)}=n^{n-1} .
$$


Multisets of unlabeled rooted trees The analog of a forest of labeled rooted trees is a multiset of unlabeled rooted trees. A multiset of unlabeled rooted trees with $m$ vertices is defined as a function $N$ from unlabeled rooted trees in $\tilde{\mathcal{A}}$ to natural numbers $\geq 0$ such that

$$
\sum_{\tau}|\tau| N(\tau)=m
$$

Thus $N(\tau)$ represents the number of times the unlabeled rooted tree $\tau$ occurs in the multiset. Every forest of labeled rooted trees gives rise to a multiset of unlabeled rooted trees, where $N(\tau)$ is the number of blocks in the forest that correspond to unlabeled rooted tree $\tau$.

Such a multiset has a symmetry factor derived from the symmetry factor associated with unlabeled rooted trees. Let $F$ be a forest with corresponding multiset $N$. The symmetry factor $\sigma(N)$ is the order of the stabilizer subgroup $\mathcal{G}_{F}$ of the forest. It is

$$
\sigma(N)=\prod_{\tau^{\prime}} N\left(\tau^{\prime}\right) ! \sigma\left(\tau^{\prime}\right)^{N\left(\tau^{\prime}\right)} .
$$

This expression may be derived using group theory. Let $\mathcal{B}$ be the subgroup of $\mathcal{G}_{F}$ generated by permutations that leave each block invariant. For each block there is a corresponding symmetry factor $\sigma\left(\tau^{\prime}\right)$, so $\mathcal{B}$ is a product group with order $\prod_{\tau^{\prime}} \sigma\left(\tau^{\prime}\right)^{N\left(\tau^{\prime}\right)}$. Let $\mathcal{H}$ be the subgroup that permutes blocks with identical unlabeled rooted trees. If unlabeled rooted tree $\tau^{\prime}$ occurs in $N\left(\tau^{\prime}\right)$ blocks, then there are $N\left(\tau^{\prime}\right)$ ! permutations involving that rooted tree. The order of $\mathcal{H}$ is thus $\prod_{\tau^{\prime}} N\left(\tau^{\prime}\right)$ !. The group $\mathcal{H}$ is a normal subgroup of $\mathcal{G}_{F}$, so in particular for every $b^{\prime}$ in $\mathcal{B}$ and for every $h$ in $\mathcal{H}$ the element $h b^{\prime} h^{-1}$ is also in $\mathcal{B}$. Every element of $\mathcal{G}_{F}$ may be uniquely expressed as product $b h$ of an element of $\mathcal{B}$ with an element of $\mathcal{H}$. (This decomposition respects multiplication: $b h b^{\prime} h^{\prime}=\left(b h b^{\prime} h^{-1}\right)\left(h h^{\prime}\right)$. In fact the group $\mathcal{G}_{F}$ is the semidirect product of the group $\mathcal{B}$ with the group $\mathcal{H}$ [3.) The conclusion is that the order of $\mathcal{G}_{F}$ is the product of the orders of $\mathcal{B}$ and $\mathcal{H}$.

Here is how to construct a forest corresponding to a given multiset $N$ with $m$ vertices. Find a set $V$ with $m$ elements and a set partition $\Gamma$ of $V$. Require that there is a function $\chi: \Gamma \rightarrow \mathcal{A}$ such that for each $\tau$ the inverse image $\chi^{-1}(\tau)$ consists of $N(\tau)$ blocks of size $|\tau|$. Finally, for each block $B$ in $\Gamma$ find a labeled rooted tree $T$ that determines unlabeled rooted tree $\chi(B)$.

The number of pairs $\Gamma, \chi$ satisfying these conditions is the coefficient

$$
C(N)=\frac{m !}{\prod_{\tau^{\prime}}\left(\left|\tau^{\prime}\right| !\right)^{N\left(\tau^{\prime}\right)}} \frac{1}{\prod_{\tau^{\prime}} N\left(\tau^{\prime}\right) !} .
$$

The first factor is the multinomial coefficient that determines how many ways of producing blocks of the appropriate sizes in a given order. The second factor has a denominator that describes how many ways there are of permuting the blocks to preserve $\chi$. The number of forests is

$$
f(N)=C(N) \prod_{\tau^{\prime}} r\left(\tau^{\prime}\right)^{N\left(\tau^{\prime}\right)}=\frac{m !}{\prod_{\tau^{\prime}} N\left(\tau^{\prime}\right) ! \sigma\left(\tau^{\prime}\right)^{N\left(\tau^{\prime}\right)}}=\frac{m !}{\sigma(N)} .
$$


The first equality comes from counting the number of ways of putting labeled rooted trees in the appropriate blocks. The second equality results from inserting $r\left(\tau^{\prime}\right)=\left|\tau^{\prime}\right| ! / \sigma\left(\tau^{\prime}\right)$.

Unlabeled rooted trees defined recursively There is a recursive definition of unlabeled rooted trees. An unlabeled rooted tree $\tau$ with $n \geq 1$ vertices is equivalent to a multiset $N$ of unlabeled rooted trees with $n-1$ vertices. This counts the unlabeled rooted subtrees that result when the root is removed. The recursion terminates with unlabeled rooted trees with one vertex; the corresponding multiset is zero.

The number $r(\tau)$ that counts labeled rooted trees satisfies the recursion

$$
r(\tau)=|\tau| f(N)=|\tau| C(N) \prod_{\tau^{\prime}} r\left(\tau^{\prime}\right)^{N\left(\tau^{\prime}\right)} .
$$

Here $\tau$ is an unlabeled rooted tree on $n$ vertices, and $N$ is the corresponding multiset on $n-1$ vertices. This is because a labeled rooted tree is determined by a root point and a forest over the remaining points.

There is also a recursion relation for the symmetry factors. If $\tau$ has $n$ vertices and its subtrees define a multiset $N$ with $n-1$ vertices.

$$
\sigma(\tau)=\sigma(N)=\prod_{\tau^{\prime} \in \tilde{\mathcal{A}}} N\left(\tau^{\prime}\right) ! \sigma\left(\tau^{\prime}\right)^{N\left(\tau^{\prime}\right)} .
$$

This recursion relation has an explicit solution. Let $\tau$ be an unlabeled rooted tree. Consider some labeling, so that there is a set $U$ of vertices and a labeled rooted tree on $U$. For each vertex $j$, consider the subtree above $j$, and let $N_{j}$ count the unlabeled rooted trees above this subtree. Then

$$
\sigma(\tau)=\prod_{j} \prod_{\tau^{\prime}} N_{j}\left(\tau^{\prime}\right) !
$$

Notation for unlabeled rooted trees The multiset notation gives a convenient way of describing unlabeled rooted trees. A tree with a single vertex is denoted 0. Otherwise, the tree is denoted $N_{1}\left[\tau_{1}\right] N_{2}\left[\tau_{2}\right] \ldots N_{k}\left[\tau_{k}\right]$, where each $N_{j} \neq 0$ and the $\tau_{j}$ are descriptions of different unlabeled rooted trees. It is convenient to abbreviate $N[0]$ by $N$. Thus, for example, the labeled rooted tree $c[b[e[a d]]$ would determine the unlabeled rooted tree $1[0] 1[2[0]]$. In the abbreviated form this would be 11[2]. This says that the root has 1 immediate successor with a single vertex and 1 immediate successor that is a tree with 2 immediate successor vertices.

Example: For $n=1$ the only rooted tree is 0, consisting of a single root point. For $n=2$ and a given vertex set there are two labeled rooted trees, depending on which point is chosen for the root. There is only one unlabeled rooted tree, denoted 1 . For $n=3$ and a given vertex set there are $3^{2}=9$ labeled rooted trees. These decompose into two orbits, as shown in Figure 1. These correspond to unlabeled rooted trees $\tau$ that may be denoted 2 and 1[1]. The rooted tree 2 has a root with two leaves. The symmetry factor is 2 . The 


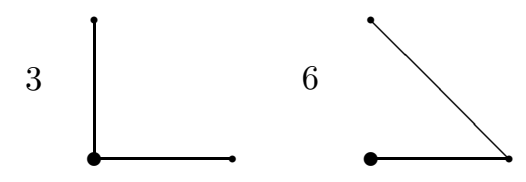

Figure 6: Unlabeled rooted trees on three vertices: $\sigma(\tau)=2,1$
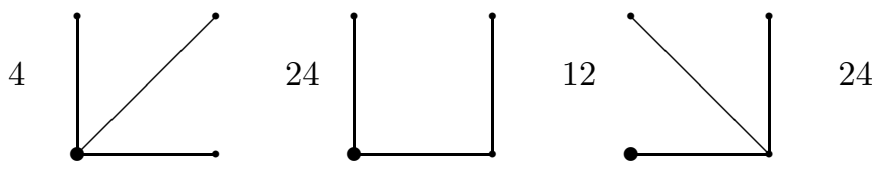

Figure 7: Unlabeled rooted trees on four vertices: $\sigma(\tau)=6,1,2,1$

1[1] linear rooted tree has a root and a successor rooted tree 1 . The symmetry factor is 1 . This gives the correct number of labeled rooted trees as the sum $6 / 2+6 / 1=9=3^{2}$. The two unlabeled rooted trees are shown in Figure 6 . \|

Example: The case $n=4$ is more interesting. There are four unlabeled rooted trees, which may be denoted in multiset notation by $3,11[1], 1[2]$, and $1[1[1]]$. These rooted trees have symmetry factors $6,1,2,1$. The number of labeled rooted trees is the sum $24 / 6+24 / 1+24 / 2+24 / 1=64=4^{3}$. See Figure 7 for a picture of the unlabeled rooted trees. II

Remark: The formula for the number $a_{n}$ of labeled rooted trees on a vertex set with $n$ vertices is $a_{n}=n^{n-1}$. The number $\tilde{a}_{n}$ of unlabeled rooted trees with $n$ vertices is not so easy to compute. This may be seen by contrasting the generating functions.

The exponential generating function for the number $a_{n}$ of labeled rooted trees with $n$ vertices is $a(t)=\sum_{n=1}^{\infty} \frac{1}{n !} a_{n} t^{n}$. The recursive definition of labeled rooted tree from root point and forest gives

$$
a(t)=t \exp (a(t))
$$

Labeled enumeration give a simple result: for fixed $t$ the value $x=a(t)$ satisfies the fixed point equation $x=t \exp (x)$.

Contrast this with the generating function for the number $\tilde{a}_{n}$ of unlabeled rooted trees with $n$ vertices. This is $\tilde{a}(t)=\sum_{n=1}^{\infty} \tilde{a}_{n} t^{n}$. The recursive definition of unlabeled rooted tree from multiset gives the identity

$$
\tilde{a}(t)=t \exp \left(\sum_{k=1}^{\infty} \frac{1}{k} \tilde{a}\left(t^{k}\right)\right)
$$

Unlabeled enumeration produces a much more complicated equation. See 2] or [1] for the full story. ॥ 


\section{Fixed point equations and labeled rooted trees}

Let $\beta(x)$ be a formal power series in $x$. Let $t$ and $g$ be parameters. Consider the fixed point equation

$$
x=g+t \beta(x) .
$$

Proposition 5 The fixed point equation $x=g+t \beta(x)$ has the formal solution

$$
x=f(t, g)=\sum_{n=0}^{\infty} \frac{t^{n}}{n !} f_{n}(g),
$$

where $f_{0}(g)=g$, and where for for $n \geq 1$ the coefficient $f_{n}(g)$ has the explicit representation

$$
f_{n}(g)=\left(\frac{\partial}{\partial g}\right)^{n-1} \beta(g)^{n}
$$

Proof: Fix $g$. The problem is to find the expansion of $x$ as a function of $t$. We know the inverse function

$$
t=(x-g) / \beta(x)
$$

giving $t$ as a function of $x$. Notice that $t=0$ corresponds to $x=g$. The Lagrange inversion formula applies. The formula is based on the the fact that the residue of a differential form expressed by a formal Laurent series is invariant under change of variable. Start with the identity

$$
\frac{1}{n} d\left(\frac{x}{t^{n}}\right)=-\frac{x}{t^{n+1}} d t+\frac{1}{n} \frac{1}{t^{n}} d x .
$$

Since the left hand is a perfect differential, it has residue zero. This gives an identity for the residues

$$
\operatorname{res}\left(\frac{x}{t^{n+1}} d t\right)=\frac{1}{n} \operatorname{res}\left(\frac{1}{t^{n}} d x\right) .
$$

This is the Lagrange inversion formula.

In the case at hand

$$
\frac{1}{n !} f_{n}(g)=\operatorname{res}\left(\frac{x}{t^{n+1}} d t\right)=\frac{1}{n} \operatorname{res}\left(\frac{1}{t^{n}} d x\right)=\frac{1}{n} \operatorname{res}\left(\frac{\beta(x)^{n}}{(x-g)^{n}} d x\right),
$$

where the last residue is computed at the singularity $x=g$. The residue is $1 /(n-1)$ ! times the $n-1$ st derivative of $\beta(g)^{n}$ with respect to $g$. This gives the result. II

A combinatorial solution gives more detailed information. This is given by an expansion indexed by rooted trees. For each $n \geq 1$ fix a label set $U_{n}$ with $n$ elements, and consider rooted trees $T$ with the label set as vertex set. For $n=0$ there is an empty set object associated with the label set $U_{0}=\emptyset$. 
Proposition 6 The fixed point equation $x=g+t \beta(x)$ has the solution given by a formal power series as above, where for $n \geq 1$ the coefficient $f_{n}(g)$ has the explicit representation

$$
f_{n}(g)=\sum_{T \in \mathcal{A}\left[U_{n}\right]} f_{T}(g)
$$

For $n \geq 1$ the coefficient

$$
f_{T}(g)=\prod_{j \in U_{n}}\left(\frac{\partial}{\partial g}\right)^{\left|T^{-1}(j)\right|} \beta(g),
$$

where $\left|T^{-1}(j)\right|$ is the degree of vertex $j$ of rooted tree $T$. For $n=0$ the contribution from the empty rooted tree is $f_{\emptyset}(g)=g$.

Proof: This proof uses calculus formula in the form explained in the appendix. Identify the $n$ factors in $\beta(g)^{n}$ with the $n$ points in the vertex set $U_{n}$. Each derivative corresponds to a point in $[1, n-1]=\{1, \ldots, n-1\}$. Use the product rule to expand

$$
\left(\frac{\partial}{\partial g}\right)^{n-1} \prod_{\ell \in U_{n}} \beta(g)=\sum_{\phi:[n-1] \rightarrow U_{n}} \prod_{\ell \in U_{n}}\left(\frac{\partial}{\partial g}\right)^{\phi^{-1}(\ell)} \beta(g) .
$$

The sum is over functions $\phi$ that pick out for each of the $n-1$ derivatives the factor to which it applies.

Use the Prüfer correspondence. Given a linear order on $U_{n}$, there is a corresponding bijection between functions $\phi$ from $[n-1]$ to $U_{n}$ and rooted trees with vertex set $U_{n}$. Furthermore, given a rooted tree $T$, the number of times the function assumes value $j$ in $U_{n}$ is the degree $\left|T^{-1}(j)\right|$. $\|$

The above may be expressed in an elegant way as

$$
f(t, g)=\sum_{T \in \mathcal{A}_{\emptyset}} \frac{1}{|T| !} t^{|T|} f_{T}(g) .
$$

Here $|T|$ denotes the number of vertices of the rooted tree, and there is one label vertex set for each value of this number.

Remark: The problem of counting labeled rooted trees is the special case when $\beta(g)=\exp (g)$. In that case $f_{n}(g)=n^{n-1} \exp (n g)$ and each $f_{T}(g)=$ $\exp (n g)$. In this special case the result is Cayley's formula $n^{n-1}=\left|\mathcal{A}\left[U_{n}\right]\right| . \quad \|$

The solution of the fixed point equation may be expressed more economically in terms of unlabeled rooted trees by

$$
f(t, g)=\sum_{\tau \in \tilde{\mathcal{A}}_{\emptyset}} \frac{r(\tau)}{|\tau| !} t^{|\tau|} f_{\tau}(g)=\sum_{\tau \in \tilde{\mathcal{A}}_{\emptyset}} \frac{1}{\sigma(\tau)} t^{|\tau|} f_{\tau}(g) .
$$

Here $r(\tau)$ is the corresponding number of labeled rooted trees, $|\tau|$ is the number of vertices, and $\sigma(\tau)$ is the symmetry factor. 
Example: Use the notation $f_{\tau}(g)$ to denote the factor associated with unlabeled rooted tree $\tau$. Then

$$
\begin{aligned}
f(x, t) & =g+f_{0} t+f_{1}(g) t^{2}+\left(\frac{1}{2} f_{2}(g)+f_{1[2]}(g)\right) t^{2} \\
+ & \left(\frac{1}{6} f_{3}(g)+f_{11[1]}(g)+\frac{1}{2} f_{1[2]}(g)+f_{1[1[1])}(g)\right) t^{4}+\cdots
\end{aligned}
$$

Explicitly, this is

$$
\begin{aligned}
& f(x, t)=g+\beta(g) t+\beta^{\prime}(g) \beta(g) t^{2}+\left(\frac{1}{2} \beta^{\prime \prime}(g) \beta(g)^{2}+\beta^{\prime}(g)^{2} \beta(g)\right) t^{3} \\
&+\left(\frac{1}{6} \beta^{\prime \prime \prime}(g) \beta(g)^{3}+\beta^{\prime \prime}(g) \beta^{\prime}(g) \beta(g)^{2}+\frac{1}{2} \beta^{\prime \prime}(g) \beta^{\prime}(g) \beta(g)^{2}+\beta^{\prime}(g)^{3} \beta(g)\right) t^{4}+\cdots
\end{aligned}
$$

To determine the contribution of a rooted tree, all that is needed is to know the number of vertices with given degree. II

\section{$5 \quad$ Increasing rooted trees}

Increasing rooted trees as partially ordered sets The convention used here is that the partial order of a labeled rooted tree increases as one moves away from the root. Consider a non-empty label set $U$ together with a given linear order $\leq_{\operatorname{lin}}$. An increasing rooted tree is a labeled rooted tree with the property that the map from $U$ with its rooted tree partial order $\leq_{\text {tree }}$ to $U$ with the given linear order $\leq_{\operatorname{lin}}$ is order-preserving. In other words, if $i \leq_{\text {tree }} j$ in the partial order of the labeled rooted tree, then the $i \leq_{\operatorname{lin}} j$ in the linear order of the labels. For an increasing rooted tree the root $r$ is the least element in both orders. The greatest element in the linear order is maximal in the partial order, so it is a leaf.

Increasing rooted trees as functions Consider a non-empty label set $U$ together with a given linear order. An increasing rooted tree is a labeled rooted tree $T: U \backslash\{r\} \rightarrow U$ that is decreasing with respect to the linear order. In other words, it is required that $T(j) \leq_{\operatorname{lin}} j$ in the linear order for all $j \neq r$. The collection of all increasing rooted trees on $U$ is denoted $\mathcal{A}^{\uparrow}[U]$.

The relation between the three kinds of rooted trees for a given linearly ordered label set $U$ with $n \geq 1$ elements is

$$
\mathcal{A}^{\uparrow}[U] \rightarrow \mathcal{A}[U] \rightarrow \tilde{\mathcal{A}}[n] .
$$

The first map is an injection, and the second map is a surjection. The composite map is also a surjection.

Increasing rooted trees defined recursively from below For each $k$ in $U$ the set of all $\ell$ with $k \leq_{\text {tree }} \ell$ is also an increasing rooted tree $T_{k}$, with root $k$. If $r$ is the root, then it must be the least element of $U$. So for each immediate successor $j$ of $r$ the rooted tree $T_{j}$ is an increasing rooted tree. This 
gives a recursive characterization of an increasing rooted tree on $U$ as a forest of increasing rooted trees on $U \backslash\{r\}$.

Increasing rooted trees defined recursively from above This gives another recursive description. Let $m$ be the greatest element of $U$ in the linear order. An increasing rooted tree on $U$ is a increasing rooted tree on $U \backslash\{m\}$ together with a point in $U \backslash\{m\}$. Thus there is an edge in the tree from $m$ to the chosen point. By taking $m=n, n-1, n-2, \ldots, 2$ this defines a map $\phi$ from $\{2,3, \ldots, n\}$ to the set of non-leaf vertices. (This is a variation on the Prüfer correspondence.)

Increasing rooted trees as permutations Each increasing rooted tree on $\{1, \ldots, n\}$ may be coded as a permutation of $\{2, \ldots, n\}$. Such a permutation may be represented as a list of the elements of $\{2, \ldots, n\}$ in some order. For $n=2$ the only entry in the list is 2 . Suppose $n \geq 3$ and an increasing rooted trees on $\{1, \ldots, n-1\}$ is coded as a list taken from $\{2, \ldots, n-1\}$. Consider a new increasing rooted tree on $\{1, \ldots, n\}$. If the tree sends $n$ to $k$ with $1 \leq k \leq n-1$, then create a new list such that for $j<k$ the $j$ th place entry is the same, the $k$ th place entry is $n$, and for $j>k$ the entry in the $j$ th place is the original $j-1$ place entry. This represents the new increasing rooted tree as a list taken from $\{2, \ldots, n\}$.

Example: The tree 1[2] is encoded by 2 . The trees $1[23]$ and $1[2[3]]$ are encoded by 32 and 23. The trees 1[234] and 1[32[4]] and 1[23[4]] are encoded by 432 and 342 and 324 , while the trees $1[42[3]]$ and $1[2[34]]$ and $1[2[3[4]]]$ are encoded by 423 and 243 and 234 . II

The permutation representation immediately gives the following result.

Proposition 7 The number of increasing rooted trees on a label set with $n$ vertices is $(n-1)$ !.

The rooted tree factorial $T$ ! of a labeled rooted tree $T$ with root $r$ is defined inductively as the number of vertices of $T$ times the product over $i$ with $T(i)=r$ of $T_{i}$ !. (An empty product gives 1.) This is an invariant under isomorphism, so for each unlabeled rooted tree $\tau$ there is a rooted tree factorial $\tau$ !. The rooted tree factorial satisfies the recursive relation

$$
\tau !=|\tau| \prod_{\tau^{\prime}}\left(\tau^{\prime} !\right)^{N\left(\tau^{\prime}\right)},
$$

where $N$ counts the subtrees obtained by removing the root.

There is another formula for the rooted tree factorial that is often convenient. For an unlabeled rooted tree $\tau$ consider a corresponding labeled rooted tree $T$. Let $T_{j}$ be the subtree over vertex $j$. Then

$$
\tau !=\prod_{j}\left|T_{j}\right|,
$$

where the product is over all vertices of $T$. The quantity $\left|T_{j}\right|$ is the number of vertices of $T_{j}$. 


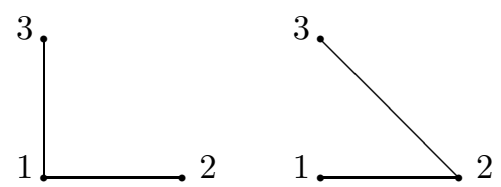

Figure 8: Increasing rooted trees on three vertices: $\tau !=3,6$

The number of increasing rooted trees per unlabeled rooted tree satisfies a recursion relation

$$
i(\tau)=C(N) \prod_{\tau^{\prime}} i\left(\tau^{\prime}\right)^{N\left(\tau^{\prime}\right)}
$$

where $N\left(\tau^{\prime}\right)$ counts immediate successor rooted trees $\tau^{\prime}$. This is similar to the formula $r(\tau)$ for the number of rooted trees per unlabeled rooted tree; the distinction is that there is only one choice of root point. It follows that the ratio $r(\tau) / i(\tau)$ satisfies

$$
\frac{r(\tau)}{i(\tau)}=|\tau| \prod_{\tau^{\prime}}\left(\frac{r\left(\tau^{\prime}\right)}{i\left(\tau^{\prime}\right)}\right)^{N\left(\tau^{\prime}\right)},
$$

where $N$ counts the successor rooted trees obtained by removing the root. This leads to the following relation.

Proposition 8 Fix $n$ and an unlabeled rooted tree $\tau$ with $n$ vertices. The ratio of the number of labeled rooted trees to the number of increasing rooted trees is the rooted tree factorial

$$
\frac{r(\tau)}{i(\tau)}=\tau !
$$

As a consequence, the number of increasing rooted trees for given unlabeled rooted tree $\tau$ is

$$
i(\tau)=\frac{r(\tau)}{\tau !}=\frac{|\tau| !}{\tau ! \sigma(\tau)}
$$

For $\tau$ with $n$ vertices there is an identity that expresses the fact that the sum over unlabeled rooted trees of the corresponding number of increasing labeled rooted trees is the total number of increasing labeled rooted trees.

Proposition 9 The sum over unlabeled rooted trees with $n$ vertices of the corresponding number of increasing rooted trees gives

$$
\sum_{\tau \in \tilde{\mathcal{A}}[n]} \frac{n !}{\sigma(\tau) \tau !}=(n-1) !
$$

Example: When $n=3$ there are only two increasing rooted trees. The symmetry factors are 2 and 1 , while the rooted tree factorials are 3 and 6 . This is illustrated in Figure 8. $\|$

Example: The case $n=4$ is more interesting. There are 3 ! $=6$ increasing rooted trees. These map to the four unlabeled rooted trees, which are 

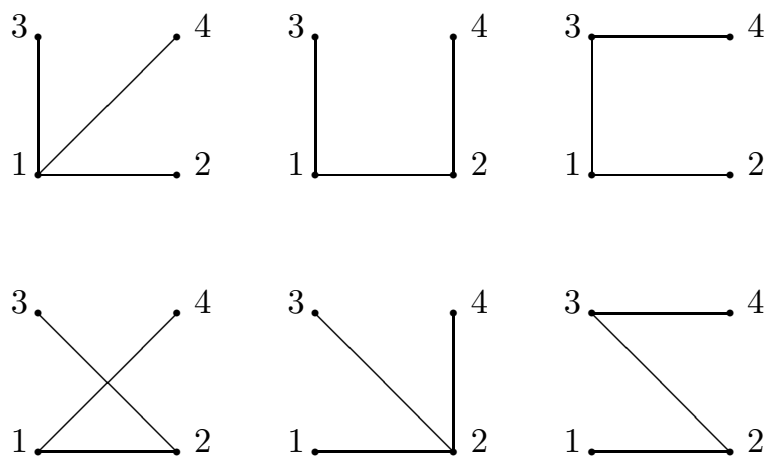

Figure 9: Increasing rooted trees on four vertices: $\tau$ ! $=4,8,8,12,24$

$3,11[1], 1[2], 1[1[1]]$. These four unlabeled rooted trees have symmetry factors $6,1,2,1$ and rooted tree factorials $4,8,12,24$. Three of the increasing rooted trees correspond to the unlabeled rooted tree 11[1] with symmetry factor 1 and rooted tree factorial 8 . The number of increasing labeled rooted trees is the sum $24 /(6 \cdot 4)+24 /(1 \cdot 8)+24 /(2 \cdot 12)+24 /(1 \cdot 24)=1+3+1+1=6$. The picture is in Figure 9. $\|$

Example: For $n=5$ vertices there are 9 unlabeled rooted trees. These are indicated in Table 1. The symmetry group of all permutations has order $n !=120$. The table lists the symmetry factors $\sigma(\tau)$, the number of labeled rooted trees $r(\tau)$, the tree factorial $\tau$ !, and the number of unlabeled rooted trees. The symmetry factor $\sigma(\tau)$ may be read off from the multiset description of $\tau$. The other quantities are related by $r(\tau)=n ! / \sigma(\tau)$ and $i(\tau)=r(\tau) / \tau$ !. If all is well, the total number of labeled rooted trees should be $n^{n-1}=625$, while the total number of increasing rooted trees should be $(n-1) !=24$.

Remark: It is instructive to look at the exponential generating function for the number $a_{n}^{\uparrow}$ of increasing rooted trees with $n$ vertices. The recursive definition gives

$$
\frac{d}{d t} a^{\uparrow}(t)=\exp \left(a^{\uparrow}(t)\right)
$$

This has the easy solution $a^{\dagger}(t)=\log \left(\frac{1}{1-t}\right)$. The details are in [1]. $\|$

\section{Ordinary differential equations and increasing rooted trees}

Let $\beta(x)$ be a formal power series in $x$. Let $t$ and $g$ be parameters. Consider the ordinary differential equation

$$
\frac{d x}{d t}=\beta(x)
$$




\begin{tabular}{lrrrr}
$\tau$ & $\sigma(\tau)$ & $r(\tau)$ & $\tau !$ & $i(\tau)$ \\
\hline 4 & 24 & 5 & 5 & 1 \\
$21[1]$ & 2 & 60 & 10 & 6 \\
$2[1]$ & 2 & 60 & 20 & 3 \\
$11[2]$ & 2 & 60 & 15 & 4 \\
$11[1[1]]$ & 1 & 120 & 30 & 4 \\
$1[3]$ & 6 & 20 & 20 & 1 \\
$1[11[1]]$ & 1 & 120 & 40 & 3 \\
$1[1[2]]$ & 2 & 60 & 60 & 1 \\
$1[1[1[1]]]$ & 1 & 120 & 120 & 1
\end{tabular}

Table 1: Unlabeled rooted trees on 5 vertices

with initial condition $x=g$ at $t=0$.

Proposition 10 This ordinary differential equation has the formal solution

$$
x=\bar{f}(t, g)=\sum_{n=0}^{\infty} \frac{t^{n}}{n !} \bar{f}_{n}(g),
$$

where the coefficient $\bar{f}_{n}(g)$ has the explicit representation

$$
\bar{f}_{n}(g)=\left(\beta(g) \frac{\partial}{\partial g}\right)^{n} g .
$$

Proof: Let the solution of the initial value problem be $\bar{f}(t, g)$. It is easy to see by induction that

$$
\frac{\partial^{n} \bar{f}(t, g)}{\partial t^{n}}=h_{n}(\bar{f}(t, g))
$$

for a suitable function $h_{n}(x)$. Taking one more derivative gives

$$
h_{n+1}(\bar{f}(t, g))=h_{n}^{\prime}(\bar{f}(t, g)) \beta(\bar{f}(t, g)) \text {. }
$$

Setting $t=0$ gives $\bar{f}(0, g)=g$, so the result is

$$
h_{n+1}(g)=h_{n}^{\prime}(g) \beta(g) .
$$

The conclusion follows immediately. \|

The combinatorial solution is given by an expansion indexed by increasing rooted trees. For each $n \geq 1$ fix a linearly ordered label set $U_{n}$ with $k$ elements. For instance, take $U_{n}=\{1, \ldots, n\}$ with the usual linear order. Furthermore, consider increasing rooted trees $\bar{T}$ with the label set as vertex set. For $n=0$ introduce an empty set object.

Proposition 11 The ordinary differential equation has the solution given by a formal power series as above, where $\bar{f}_{n}(g)$ has the explicit representation

$$
\bar{f}_{n}(g)=\sum_{\bar{T} \in \mathcal{A}^{\uparrow}\left[U_{n}\right]} f_{\bar{T}}(g)
$$


For $n \geq 1$ the coefficient is

$$
f_{\bar{T}}(g)=\prod_{j \in U_{n}}\left(\frac{\partial}{\partial g}\right)^{\left|\bar{T}^{-1}(j)\right|} \beta(g),
$$

where $\left|\bar{T}^{-1}(j)\right|$ is the degree of vertex $j$ of rooted tree $\bar{T}$. For $n=0$ the contribution from the empty rooted tree is $f_{\emptyset}(g)=g$.

Proof: Write

$$
\bar{f}_{n}(g)=\left(\beta(g) \frac{\partial}{\partial g}\right)^{n-1} \beta(g) .
$$

Index the partial derivatives from $n$ down to 2 . Index the $\beta(g)$ factors from $n$ down to 1 . Then every partial derivative acts only on the $\beta(g)$ factors with strictly smaller index. So

$$
\bar{f}_{n}(g)=\sum_{\phi} \prod_{j=1}^{n}\left(\frac{\partial}{\partial g}\right)^{\left|\phi^{-1}(j)\right|} \beta(g),
$$

where the sum is over functions $\phi$ from to $[2, n]$ to $[1, n]$ with the property that $\phi(i)<i$ for all $i$.

Every such function $\phi$ is an increasing rooted tree on $[1, n]$, where $\phi(i)$ is the immediate predecessor of $i$, and $\phi^{-1}(j)$ is the set of immediate successors of $j$. II

The above may be expressed in an elegant way as

$$
\bar{f}(t, g)=\sum_{\bar{T} \in \mathcal{A}_{\emptyset}^{\uparrow}} \frac{1}{|\bar{T}| !} t^{|\bar{T}|} f_{\bar{T}}(g) .
$$

Here $|\bar{T}|$ denotes the number of vertices of the increasing rooted tree, and there is one label vertex set for each value of this number.

Remark: The problem of counting increasing rooted trees is the special case when $\beta(g)=\exp (g)$. The solution of the differential equation is given explicitly by $x=g-\log (1-\exp (g) t)$. The coefficient in $n$th order is $\bar{f}_{n}(g)=$ $(n-1) ! \exp (n g)$, and each $f_{T}(g)=\exp (n g)$. In this special case the result is equivalent to the formula $(n-1) !=\left|\mathcal{A}^{\uparrow}\left[U_{n}\right]\right|$. $\quad \mid$

The solution of the ordinary differential equation may be expressed in terms of unlabeled rooted trees by

$$
\bar{f}(t, g)=\sum_{\tau \in \tilde{\mathcal{A}}_{\emptyset}} \frac{i(\tau)}{|\tau| !} t^{|\tau|} f_{\tau}(g)=\sum_{\tau \in \tilde{\mathcal{A}}_{\emptyset}} \frac{1}{\sigma(\tau) \tau !} t^{|\tau|} f_{\tau}(g) .
$$

Here $r(\tau)$ is the corresponding number of increasing rooted trees, $|\tau|$ denotes the number of vertices of the rooted tree, $\sigma(\tau)$ is the symmetry factor, and $\tau$ ! is the rooted tree factorial. 
Example: Use the notation $f_{\tau}(g)$ to denote the factor associated with unlabeled rooted tree $\tau$. Then

$$
\begin{aligned}
& \bar{f}(x, t)=g+f_{0} t+\frac{1}{2} f_{1}(g) t^{2}+\frac{1}{6}\left(f_{2}(g)+f_{1[2]}(g)\right) t^{2} \\
& +\frac{1}{24}\left(f_{3}(g)+3 f_{11[1]}(g)+f_{1[2]}(g)+f_{1[1[1]]}(g)\right) t^{4}+\cdots .
\end{aligned}
$$

Explicitly, this is

$$
\begin{gathered}
\bar{f}(x, t)=g+\beta(g) t+\frac{1}{2} \beta^{\prime}(g) \beta(g) t^{2}+\frac{1}{6}\left(\beta^{\prime \prime}(g) \beta(g)^{2}+\beta^{\prime}(g)^{2} \beta(g)\right) t^{3} \\
+\frac{1}{24}\left(\beta^{\prime \prime \prime}(g) \beta(g)^{3}+3 \beta^{\prime \prime}(g) \beta^{\prime}(g) \beta(g)^{2}+\beta^{\prime \prime}(g) \beta^{\prime}(g) \beta(g)^{2}+\beta^{\prime}(g)^{3} \beta(g)\right) t^{4}+\cdots
\end{gathered}
$$

The factor 3 comes from the 3 increasing rooted trees associated with $\tau=11(1)$. These ordinary differential equation coefficients are the fixed point equation coefficients divided by tree factorials. \|

\section{The Butcher group (composition) for labeled rooted trees}

Let $T$ be a labeled rooted tree with vertex set $U$ and root $r$ in $U$. A rooted subtree $T_{0}$ is a rooted tree on some non-empty subset $U_{0} \subseteq U$ with the same root $r$ in $U_{0}$ that is a restriction of $T$ to this subset. The condition that $T_{0}$ is a rooted subtree of $T$ is denoted $T_{0} \rightarrow T$. The empty subset $U_{0}=\emptyset$ corresponds to an empty set object $T_{0}$; that case is also abbreviated $T_{0} \rightarrow T$.

For each $T_{0}$ with $T_{0} \rightarrow T$ there is a corresponding difference forest $T \backslash T_{0}$ of rooted trees on $U \backslash U_{0}$. The trees in the forest are all subtrees $T_{j}$ with $j \in U \backslash U_{0}$ and $T(j) \in U_{0}$. If $U_{0}=\emptyset$ and $T_{0}$ is the empty set object, then the difference forest consists of the tree $T$ on $U$.

Proposition 12 For each non-empty label set $U$ there is a one-to-one correspondence between rooted tree pairs $T_{0}, T$ with $T_{0} \rightarrow T$ and triples $T_{0}, F_{1}, \phi$, where $T_{0}$ is a rooted tree on $U_{0} \subseteq U, F_{1}$ is a forest on $U_{1}=U \backslash U_{0}$, and $\phi$ is a function from the set partition $\Gamma_{1}$ of the forest to $U_{0}$.

Let $\tilde{\mathcal{A}}_{\emptyset}$ be the set of unlabeled rooted trees together with the empty set object. Consider the space $\mathbf{R}^{\tilde{\mathcal{A}}_{\emptyset}}$ of all functions $c$ from $\tilde{\mathcal{A}}_{\emptyset}$ to the real numbers. These are coefficients $c(\tau)$ that depend on unlabeled rooted trees $\tau$. Since each labeled rooted tree $T$ determines a corresponding unlabeled rooted tree $\tau$, the coefficients $c(T)$ are also defined for labeled rooted trees. For a forest of rooted trees the coefficient $a^{\times}(F)$ is the product of the $a\left(T^{\prime}\right)$ for $T^{\prime}$ in the forest. In particular, for the empty forest $a^{\times}(\emptyset)=1$.

The operation of subtree convolution $a * b$ is defined when $a(\emptyset)=1$ by $c=a * b$, where

$$
c(T)=\sum_{T_{0}: T_{0} \rightarrow T} b\left(T_{0}\right) a^{\times}\left(T \backslash T_{0}\right) .
$$


When $T_{0}$ is the empty set object, the corresponding term in the sum is is $b(\emptyset) a(T)$. When $T_{0}=T$, the corresponding term in the sum is $b(T) a^{\times}(\emptyset)=$ $b(T)$. When $T$ is the empty set object, then $c(\emptyset)=b(\emptyset)$.

In the multiplication $a * b$ the forest factor is on the left and the tree factor is on the right. This convention is common in this context 14,8 . The Butcher group multiplication is the special case when both $a(\emptyset)=1$ and $b(\emptyset)=1$. This group is denoted $G_{C}$, where $C$ stands for composition. The identity $\delta_{\emptyset}$ in the group has coefficient 1 for the empty set object and 0 for the rooted trees. An inductive argument shows that every element has an inverse element. The group $G_{C}$ is the character group of the rooted tree Hopf algebra of Connes and Kreimer.

Example: It is easy to compute the Butcher group multiplication for reasonably small labeled rooted trees. Here are the results for up to 3 vertices. For notational simplicity label the tree as an increasing tree. Take the root with label 1. For one-vertex rooted trees $c(1)=b(1)+a(1)$. For two-vertex rooted trees the result is $c(1[2])=b(1[2])+b(1) a(2)+a(1[2])$. The first interesting case is $n=3$. Let $1[23]$ be the rooted tree with root at 1 and with leaves at 2 and 3 , and let $1[2[3]]$ be the rooted tree with root at 1 and with successor rooted tree $2[3]$. Then

$$
c(1[23])=b(1[23])+b(1[2]) a(3)+b(1[3]) a(2)+b(1) a(2) a(3)+a(1[23]) .
$$

Similarly

$$
c(1[2[3]])=b(1[2[3]])+b(1[2]) a(3)+b(1) a(2[3])+a(1[2[3]]) .
$$

The expressions for the two cases are quite different. II

Example: Here is one calculation for $n=4$. The rooted tree is $T=1[3[2[4]]$ with root at 1 and successor rooted trees 3 and 2[4]. This happens to be an increasing rooted tree, but that is just for notational convenience. The result is

$$
\begin{aligned}
& c(T)=b(T)+b(1[2[4]]) a(3)+b(1[23]) a(4) \\
& \quad+b(1[3]) a(2[4])+b(1[2]) a(3) a(4))+b(1) a(2[4]) a(3)+a(T) .
\end{aligned}
$$

This is illustrated in Figure 10. The number of vertices in the subtrees are 4, 3, $3,2,2,1,0$. The difference forests consist of $0,1,1,1,2,2,1$ rooted trees. II

Coefficients depending on rooted trees also define certain functions (defined as formal power series). Each such function is a weighted tree sum in the form of an exponential generating function. For coefficients $c$ the function is

$$
f^{c}(t, g)=\sum_{T \in \mathcal{A}_{\emptyset}} \frac{1}{|T| !} t^{|T|} c(T) f_{T}(g) .
$$

Again for rooted tree $T$ the coefficient is

$$
f_{T}(g)=\prod_{\ell \in U}\left(\frac{\partial}{\partial g}\right)^{\left|T^{-1}(\ell)\right|} \beta(g) .
$$



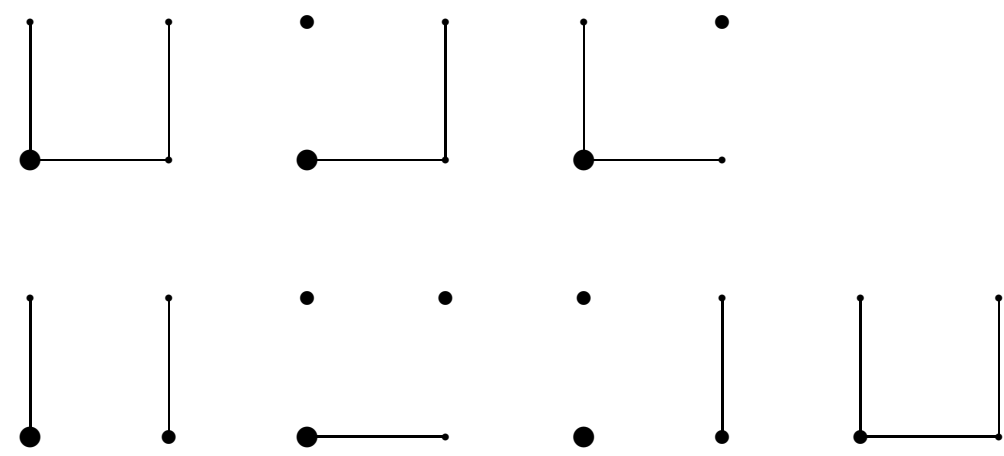

Figure 10: Subtrees and difference forests

The zero order term is $c(\emptyset) g$. The remainder of the series is a power series in powers of $t$ and powers of derivatives of $\beta(g)$. Such as series may be further expanded in powers of $g$, but that is not done here.

Theorem 1 (Composition) Suppose $a(\emptyset)=1$, and let $c=a * b$ be the subtree convolution. The corresponding weighted sums are related by

$$
f^{c}(t, g)=f^{b}\left(t, f^{a}(t, g)\right) .
$$

Proof: The proof is based on the calculus formulas in the appendix. The task is to show that $f^{c}(t, g)$ and $f^{b}\left(t, f^{a}(t, g)\right)$ have the same terms of each order. The $n$th term is the $n$th partial derivative with respect to $t$, evaluated at zero. (The case $n=0$ is trivial, so assume $n \geq 1$.) Take a set $U$ with $|U|=n$ elements. For $f^{c}(t, g)$

$$
\left.D_{t}^{|U|} f^{c}(t, g)\right|_{t=0}=\sum_{T \in \mathcal{A}[U]} c(T) f_{T}(g) .
$$

Product rule The composition

$$
f^{b}\left(t, f^{a}(t, g)\right)=\sum_{T \in \mathcal{A}_{\emptyset}} \frac{1}{|T| !} t^{|T|} b(T) f_{T}\left(f^{a}(t, g)\right) .
$$

is a combination of products of two factors $t^{|T|}$ and $f_{T}\left(f^{a}(t, g)\right)$ By the product rule, the $n$th derivative is a sum over disjoint unions $U=U_{0}+U_{1}$ of the form

$$
\left.D_{t}^{|U|} f^{b}\left(t, f^{a}(t, g)\right)\right|_{t=0}=\left.\sum_{U=U_{0}+U_{1}} \sum_{T_{0} \in \mathcal{A}\left[U_{0}\right]} b\left(T_{0}\right) D_{t}^{\left|U_{1}\right|} f_{T_{0}}\left(f^{a}(t, g)\right)\right|_{t=0} .
$$


Chain rule The next task is to evaluate $D^{\left|U_{1}\right|} f_{T_{0}}\left(f^{a}(t, g)\right)$ by the chain rule. This is a sum over set partitions $\Gamma_{1}$ of $U_{1}$

$$
\left.D_{t}^{\left|U_{1}\right|} f_{T_{0}}\left(f^{a}(t, g)\right)\right|_{t=0}=\left.\left.\sum_{\Gamma_{1} \in \operatorname{Part}\left[U_{1}\right]} D_{g}^{\left|\Gamma_{1}\right|} f_{T_{0}}\left(f^{a}(t, g)\right)\right|_{t=0} \prod_{B \in \Gamma_{1}} D^{|B|} f^{a}(t, g)\right|_{t=0} .
$$

Since $f^{a}(0, g)=g$, this is

$$
\left.D_{t}^{\left|U_{1}\right|} f_{T_{0}}\left(f^{a}(t, g)\right)\right|_{t=0}=\sum_{\Gamma_{1} \in \operatorname{Part}\left[U_{1}\right]} D_{g}^{\left|\Gamma_{1}\right|} f_{T_{0}}(g) \prod_{B \in \Gamma_{1}}\left(\sum_{H \in \mathcal{A}[B]} a(H) f_{H}(g)\right) .
$$

Distributive law The distributive law is used to expand the product

$$
\prod_{B \in \Gamma_{1}}\left(\sum_{H \in \mathcal{A}[B]} a(H) f_{H}(g)\right)=\sum_{F} \prod_{B \in \Gamma_{1}} a(F(B)) f_{F(B)}(g),
$$

where $F$ is a function defined on $\Gamma_{1}$ such that the value of $F$ on block $B$ in $\Gamma_{1}$ is a rooted tree on $B$.

Product rule When $T_{0}$ is non-empty the coefficient $f_{T_{0}}(g)$ is a product over vertices $\ell$ in $U_{0}$ of factors $\beta^{(d(\ell))}(g)$. By the product rule the derivative of order $\left|\Gamma_{1}\right|$ is a sum over functions $\phi: \Gamma_{1} \rightarrow U_{0}$ in the form

$$
D_{g}^{\left|\Gamma_{1}\right|} f_{T_{0}}(g)=\sum_{\phi: \Gamma_{1} \rightarrow U_{0}} f_{T_{0}, \phi}(g)
$$

Here

$$
f_{T_{0}, \phi}(g)=\prod_{\ell \in U_{0}} \beta^{\left(\left|T_{0}^{-1}(\ell)\right|+\left|\phi^{-1}(\ell)\right|\right)}(g) .
$$

For the empty rooted tree $f_{\emptyset}(g)=g$ and so the only derivative that is non-zero is the first derivative, corresponding to a set partition of $U=U_{1}$ into one block.

It is convenient to define $f_{\emptyset, \phi}(g)=1$, where $\phi$ is some unique object whose nature is not important.

Construction of rooted tree and subtree The end result is

$$
\sum_{U=U_{0}+U_{1}}^{D_{t}^{|U|}} \sum_{T_{0} \in \mathcal{A}\left[U_{0}\right]} \sum_{\Gamma_{1} \in \operatorname{Part}\left[U_{1}\right]} \sum_{\phi: \Gamma_{1} \rightarrow U_{0}} \sum_{F} \prod_{B \in \Gamma_{1}} b\left(T_{0}\right) a(F(B)) f_{T_{0}, \phi}(g) f_{F(B)}(g) .
$$

Fix $U=U_{0}+U_{1}$ and rooted tree $T_{0}$ on $U_{0}$. The remaining data $\Gamma_{1}, \phi, F$ determine a rooted tree $T$ on $U$ that extends $T_{0}$. The value $\phi(B)$ may be thought of as the vertex to which the root of the tree $F(B)$ maps. So

$$
\left.D_{t}^{|U|} f^{b}\left(t, f^{a}(t, g)\right)\right|_{t=0}=\sum_{U=U_{0}+U_{1}} \sum_{T_{0} \in \mathcal{A}\left[U_{0}\right]} \sum_{T: T_{0} \rightarrow T} b\left(T_{0}\right) a^{\times}\left(T \backslash T_{0}\right) f_{T}(g) .
$$


The sum may be done in the other order, first the rooted tree $T$ and then the subtree $T_{0}$. This gives

$$
\left.D_{t}^{|U|} f^{b}\left(t, f^{a}(t, g)\right)\right|_{t=0}=\sum_{T \in \mathcal{A}[U]} \sum_{T_{0}: T_{0} \rightarrow T} b\left(T_{0}\right) a^{\times}\left(T \backslash T_{0}\right) f_{T}(g) .
$$

In other words, the derivative is $\sum_{T \in \mathcal{A}[U]}(a * b)(T) f_{T}(g)$. $\|$

One special case of the multiplication law is when the sequence $b$ is zero except for $b(\bullet)=1$. This corresponds to the function $f^{b}(t, g)=t \beta(g)$. In this case $c(T)=a^{\times}(T \backslash \bullet)$, the product of $a\left(T^{\prime}\right)$ for all rooted trees $T^{\prime}$ in the successor forest.

Example: Consider $f^{b}(t, g)=t \beta(g)$ and $f^{a}(t, g)=g+a(\bullet) t \beta(g)$. The composition is

$$
f^{c}(t, g)=t \beta(g+a(\bullet) t \beta(g)) .
$$

This type of composition occurs in numerical methods for the solution of ordinary differential equations. In this case $c(T)$ is zero unless $T$ is a rooted tree on a set with $n \geq 1$ points, one root and $n-1$ leaves. There are $n$ such rooted trees. The corresponding successor forests each consist of $n-1$ one point rooted trees. This gives $c(T)=a(\bullet)^{n-1}$. The function $f^{c}(t, g)$ has the rooted tree expansion

$$
f^{c}(t, g)=\sum_{n=1}^{\infty} n \frac{t^{n}}{n !} a(\bullet)^{n-1} \beta^{(n-1)}(g) \beta(g)^{n-1} .
$$

This is the Taylor expansion of the composite function.

This example underpins the Runge-Kutta methods for the numerical solution of ordinary differential equations. The first order Euler method is to use $g+$ $\beta(g) t$ to approximate the solution. Various second order methods depend on a parameter $a$; they are of the form $g+\left(1-\frac{1}{2 a}\right) \beta(g) t+\frac{1}{2 a} \beta(g+a \beta(g) t)$. In particular, $a=1$ is the analog of the trapezoidal rule, and $a=\frac{1}{2}$ is the analog of the midpoint rule. Since $\beta(g+a \beta(g) t)$ agrees with $\beta(g)+a \beta^{\prime}(g) \beta(t) t$ to second order, the second order Runge-Kutta method agrees with the Taylor method $g+\beta(t) t+\frac{1}{2} \beta^{\prime}(g) \beta(g) t$ to second order. The Runge-Kutta method has the advantage that it does not require computing the derivative $\beta^{\prime}(g)$. II

The Butcher group is related to composition of power series. Take the case when $\beta(g)=\exp (g)$. In that case the contribution of a labeled rooted tree on $n \geq 1$ vertices is $\exp (n g)$. Suppose $c=a * b$ and $f^{c}(t, g)=f^{b}\left(t, f^{a}(t, g)\right)$. Each of the individual functions is a power series in powers of $\exp (g)$. Define $h^{a}(w)=\exp \left(f^{a}(1, \log (w))\right)$ and similarly for the others. The resulting functions are power series in $w$, and they are related by $h^{c}(w)=h^{b}\left(h^{a}(w)\right)$. Subtree convolution is mapped to composition of power series. 


\section{The Butcher group for increasing rooted trees}

The composition law for the Butcher group may also be written in terms of increasing rooted trees. The function corresponding to sequence $a$ is

$$
f^{a}(t, g)=\sum_{\bar{T} \in \mathcal{A}_{\emptyset}^{\uparrow}} \frac{t^{|\bar{T}|}}{|\bar{T}| !} a(\bar{T}) \bar{T} ! f_{\bar{T}}(g) .
$$

The rooted subtree convolution is defined in the same way, since if $T$ is an increasing rooted tree and $T_{0} \rightarrow T$, then the subtree $T_{0}$ is also increasing.

If $T_{0} \rightarrow T$, the rooted tree binomial coefficient is

$$
\left(\begin{array}{c}
T \\
T_{0}
\end{array}\right)=\frac{T !}{T_{0} ! \prod_{T^{\prime} \in T \backslash T_{0}} T^{\prime} !} .
$$

For a linear rooted tree this is the usual binomial coefficient.

The change of variable $\bar{c}_{T}=c(T) T$ ! gives another representation of the Butcher multiplication as

$$
\bar{c}(T)=\sum_{T_{0}: T_{0} \rightarrow T}\left(\begin{array}{c}
T \\
T_{0}
\end{array}\right) \bar{b}\left(T_{0}\right) \bar{a}^{\times}\left(T \backslash T_{0}\right) .
$$

The solution $\bar{f}(t, g)$ of the differential equation $d x / d t=\beta(x)$ with initial condition $g$ is the case corresponding to $a(T)=1 / T$ !, or $\bar{a}(T)=1$. This fact leads to an identity for a binomial coefficient associated with rooted trees.

Proposition 13 For a labeled rooted tree with $n$ vertices

$$
\sum_{T_{0} \rightarrow T}\left(\begin{array}{c}
T \\
T_{0}
\end{array}\right)=2^{n} .
$$

Proof: The solution of the differential equation has the group property $\bar{f}(2 t, g)=\bar{f}(t, \bar{f}(t, g))$. Take $\bar{a}(T)=1$ and the corresponding $\bar{f}^{a}(t, g)$. Then $\bar{f}^{a}(2 t, g)=\bar{f}^{a}\left(t, \bar{f}^{a}(t, g)\right)$. Now take $\bar{a}(T)=\bar{b}(T)=1$, so $\bar{c}(T)=2^{|T|}$. This then translates to $\bar{f}^{c}(T)=\bar{f}^{b}\left(t, \bar{f}^{a}(t, g)\right)$. This gives the rooted tree binomial coefficient identity for increasing rooted trees. Since rooted tree factorials do not depend on the order on the label set, the identity holds for all labeled rooted trees. II

Example: For the labeled rooted tree with four vertices shown in Figure 10 the binomial identity says

$$
16=1+\frac{4}{3}+\frac{8}{3}+2+4+4+1 .
$$

The rooted tree binomial coefficients need not be whole numbers, but the sum is always a power of 2 . $\|$ 


\section{The Butcher group for unlabeled rooted trees}

The Butcher group may also be presented using unlabeled rooted trees, but there is a complication. If $\tau, \tau_{0}$ are unlabeled rooted trees, choose $T$ to be a labeled rooted tree that determines $\tau$. Consider the set of labeled rooted trees $T_{0}$ with $T_{0} \rightarrow T$ and with $T_{0}$ determining $\tau_{0}$. This set depends on the chosen $T$, but the number of elements in the set is independent of $T$. Denote this number by $\left[\tau, \tau_{0}\right]$.

The multiplication operation for coefficients may then be written $c=a * b$, where

$$
c(\tau)=\sum_{\tau_{0}}\left[\tau, \tau_{0}\right] b\left(\tau_{0}\right) a^{\times}\left(\tau \backslash \tau_{0}\right) .
$$

The extra complication is the presence of the multiplicity coefficients $\left[\tau, \tau_{0}\right]$.

For an arbitrary coefficient function $c$ there is a corresponding function

$$
f^{c}(t, g)=\sum_{\tau \in \tilde{\mathcal{A}}_{\emptyset}} \frac{1}{\sigma(\tau)} c(\tau) t^{|\tau|} f_{\tau}(g) .
$$

The zero order term is $c(\emptyset) g$, and for the other terms

$$
f_{\tau}(g)=\prod_{k}\left(D_{g}^{k} \beta(g)\right)^{v_{k}(\tau)} .
$$

This is precisely the same sum as before. The following is a restatement of the previous theorem.

Corollary 1 Suppose $a(\emptyset)=1$. Define $c=a * b$ with the multiplicity factor. The corresponding functions satisfy

$$
f^{c}(t, g)=f^{b}\left(t, f^{a}(t, g)\right) .
$$

Example: Take $k=4$ vertices, and let 3 be the unlabeled rooted tree with a root and three leaves. Let 2 be the unlabeled rooted tree with a root and two leaves. Then the multiplicity $[3,2]=3$. Similarly, $[3,1]=3$. On the other hand, $[3,0]=1$, since there is only one way of inserting the root. The conclusion is that

$$
c(3)=b(3)+3 b(2) a(0)+3 b(1) a(0)^{2}+b(0) a(0)^{3}+a(3) .
$$

In the world of unlabeled rooted trees, multiplicity factors are inescapable. ॥

\section{Substitution for labeled rooted trees}

The Butcher group is about subtree convolution and composition of functions. There is another algebraic structure based on quotient tree convolution and substitution. The reference 8 , has an account of the subject and its history, along with some applications. See also [7] for a Hopf algebra approach and for 


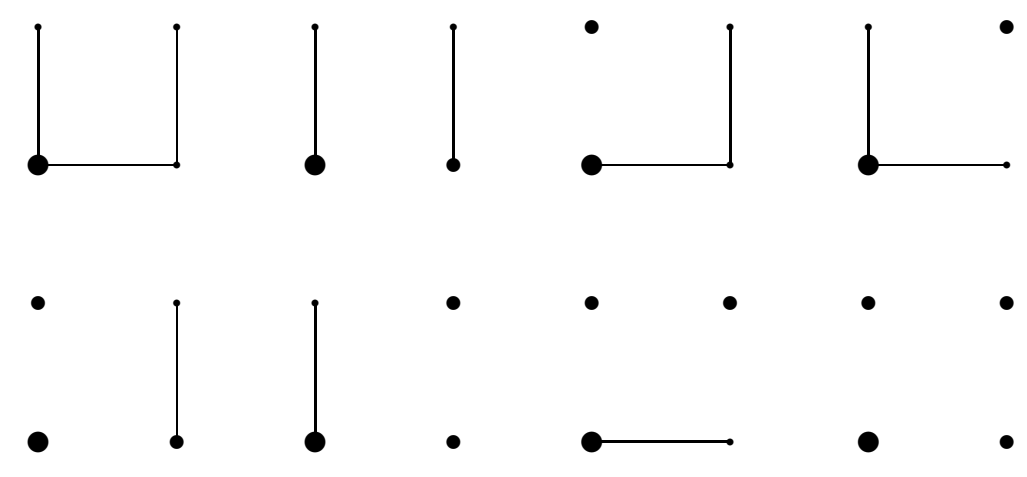

Figure 11: Subforests

more background. The following is a brief account in the labeled rooted tree framework.

Given a rooted tree $T$ on $U$ with root $r$ and a set $R$ with $r \in R$, there is a corresponding forest $F$ obtained by restricting $T$ to $U \backslash R$. This is called a subforest of the rooted tree. The set partition $\Gamma$ defined by this forest is in one-to-one correspondence with the set of roots $R$ of the rooted trees in the forest. When $F$ is a subforest of $T$ we can write $F \sqsubseteq T$.

Given a rooted tree $T$ and a subforest $F$, there is also a quotient rooted tree $T / F$. This is a labeled rooted tree with label set $\Gamma$. Let $U_{0}$ be the block in $\Gamma$ such that $r$ is in $F\left(U_{0}\right)$. Then $T / F$ is defined on $\Gamma_{1}=\Gamma \backslash\left\{U_{0}\right\}$ as follows. The value of $T / F$ on block $B$ is obtained by finding the root $j$ of rooted tree $F[B]$ and taking the value to be the block $B^{\prime}$ containing $T(j)$.

There is another representation of the quotient rooted tree $T / F$ that may be easier to picture. This is as a labeled rooted tree with label set $R$, where $R$ is the set of roots in the forest $F$. The value of $T / F$ on vertex $j$ in $R \backslash\{r\}$ is obtained by finding $T(j)$ and the block $B^{\prime}$ that contains it, and taking the value to be the root of rooted tree $F\left[B^{\prime}\right]$.

Remark: The subtree $T_{0}$ and difference forest $T \backslash T_{0}$ construction used for the Butcher group is a special case. The subforest $F$ is $T_{0}$ together with $F_{1}=T \backslash T_{0}$, and in this case the quotient rooted tree consists only of a root and immediate successors. II

Example: Figure 11 gives an example of the forests associated with a given rooted tree. The number of rooted trees in the forest (and the number of roots of these rooted trees) are 1, 2, 2, 2, 3, 3, 3, 4. Figure 12 gives an example of quotient rooted trees of a given rooted tree on 4 vertices. These correspond to the subforests in Figure 11. The roots of the rooted trees in the forest give the vertices of the quotient rooted tree. The number of vertices in the quotient rooted trees are $1,2,2,2,3,3,3,4$. $\|$

The pair consisting of the subforest $F$ and the quotient rooted tree $T / F$ do 


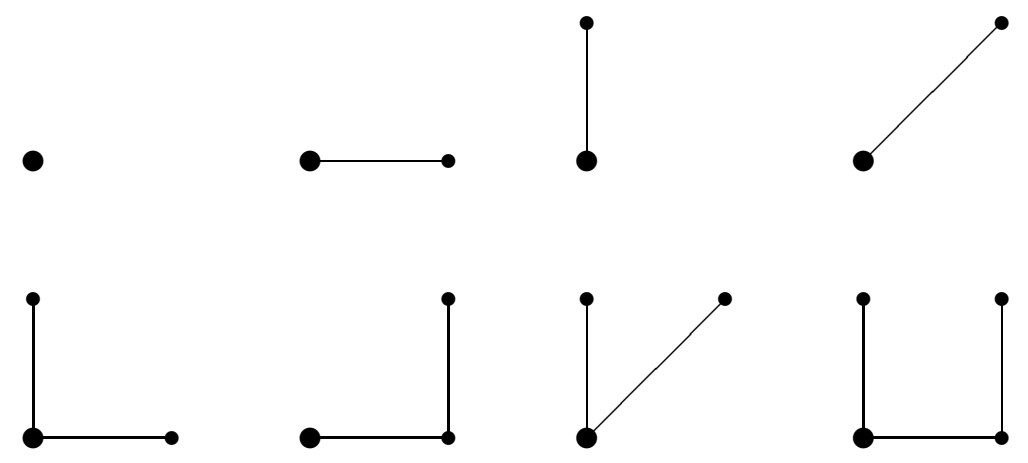

Figure 12: Quotient rooted trees

not completely determine the original rooted tree $T$. The quotient rooted tree assigns to each block in $\Gamma_{1}$ another block in $\Gamma$. It is also necessary to specify a point in that block.

Proposition 14 For each label set $U$ there is a one-to-one correspondence between rooted tree, subforest pairs $T, F$ and triples $F, \hat{T}, \phi$, where $F$ is a forest of rooted trees with set partition $\Gamma, \hat{T}$ is a rooted tree on vertex set $\Gamma$ with root $U_{0}$, and $\phi$ is a function defined on $\Gamma_{1}=\Gamma \backslash\left\{U_{0}\right\}$ such that for every $B$ in $\Gamma_{1}$ the value $\phi(B)$ is in $\hat{T}(B)$.

In the proposition the function $\phi$ sends a block to a vertex in another target block. There is a parametrization of such functions by target blocks. If $B^{\prime}$ is a block in $\Gamma$, then define $\Phi\left(B^{\prime}\right)$ to be the restriction of $\phi$ to $T^{-1}\left(B^{\prime}\right)$. Thus if $\hat{T}(B)=B^{\prime}$, then $\Phi\left(B^{\prime}\right)$ applied to $B$ is a vertex in $B^{\prime}$. Conversely, suppose that there is a function $\Phi$ that maps each $B^{\prime}$ in $\Gamma$ to a function $\Phi\left(B^{\prime}\right)$ from $\hat{T}^{-1}\left(B^{\prime}\right)$ to $B^{\prime}$. Then there is a corresponding $\phi$ given on $B$ with $\hat{T}(B)=B^{\prime}$ by $\phi(B)=\Phi\left(B^{\prime}\right)(B)$.

Let $\tilde{A}_{\emptyset}$ be the set of unlabeled rooted trees augmented with the empty object. There is a multiplication defined on certain elements of $\mathbf{R}^{\tilde{\mathcal{A}}_{\emptyset}}$. This is the quotient rooted tree convolution $c=a \star b$, defined whenever $a(\emptyset)=0$, such that

$$
c(T)=\sum_{F \sqsubseteq T} b(T / F) a^{\times}(F) .
$$

The sum is over subforests $F$ of $T$. The contribution of a forest is $a^{\times}(F)=$ $\prod_{T^{\prime} \in F} a\left(T^{\prime}\right)$. For the empty forest this product is 1 . For the special case of the empty set object $c(\emptyset)=b(\emptyset) a^{\times}(\emptyset)=b(\emptyset)$.

If $R=\{r\}$, then the forest $F$ has only one rooted tree $T$, and $T / F$ is a rooted tree on a one point vertex set. So the contribution to the sum is $a(T) b(\bullet)$. 
When $R$ is the entire vertex set, then $F$ is the discrete forest, and $T / F=T$. The contribution to the sum is $a(\bullet)^{|T|} b(T)$. As a special case, $c(\bullet)=a(\bullet) b(\bullet)$.

If the multiplication is restricted to $a(\emptyset)=b(\emptyset)=0$, then $G_{S}=\mathbf{R}^{\tilde{\mathcal{A}}}$ becomes an algebraic system closed under multiplication. The $S$ stands for substitution. The identity for quotient rooted tree convolution is $\delta_{\bullet}$, which has coefficient 1 for a one-vertex rooted tree and 0 for all other rooted trees. If the multiplication is also restricted to $a(\bullet) \neq 0, b(\bullet) \neq 0$, the resulting system $G_{S}^{\star}$ is a group. If the multiplication is further restricted to $a(\bullet)=b(\bullet)=1$, then this defines a subgroup $G_{S}^{1}$. The group $G_{S}^{1}$ is the character group of the rooted tree Hopf algebra of Calaque, Ebrahimi-Fard, and Manchon.

There is also a functional representation of quotient rooted tree convolution. This deals with formal functions of the form $g \mapsto \alpha(g)=\sum_{n=0}^{\infty}(1 / n !) a_{n} g^{n}$. Such a function is denoted $\alpha$ to indicate that it depends only on the coefficients $a_{n}$ and not on the input $g$. The power series depends on the choice of $\alpha$ and is of the form

$$
f^{c}(t, \alpha, g)=\sum_{n=0}^{\infty} \sum_{T \in \mathcal{A}\left[U_{n}\right]} \frac{t^{n}}{n !} c(T) \prod_{\ell \in U_{n}} \alpha^{\left(\left|T^{-1}(\ell)\right|\right)}(g) .
$$

The zero order term is $c(0) g$. This leads to the following theorem $[8]$.

Theorem 2 (Substitution) Suppose that $a(\emptyset)=0$ and $c=a \star b$ is the quotient rooted tree convolution. Then

$$
f^{c}(t, \beta, g)=f^{b}\left(1, f^{a}(t, \beta, \cdot), g\right) .
$$

Proof: The proof here uses the calculus formulas in the appendix. Let $D_{t}$ be the partial derivative with respect to $t$. It is sufficient to show that applying $D_{t}^{n}$ and then setting $t$ to zero gives the same result for both sides of the equation. For the left hand side this is

$$
\left.D_{t}^{|U|} f^{c}(t, \beta, g)\right|_{t=0}=\sum_{T \in \mathcal{A}[U]} c(T) \prod_{\ell \in U} D_{g}^{\left|T^{-1}(\ell)\right|} \beta(g),
$$

where $U$ is a label set with $n$ points.

Product rule The computation for the right hand side begins with

$$
f^{b}\left(1, f^{a}(t, \beta, \cdot), g\right)=\sum_{\hat{T}} \frac{1}{|\hat{T}| !} b(\hat{T}) \prod_{i \in[\hat{T}]} D_{g}^{\left|\hat{T}^{-1}(i)\right|} f^{a}(t, \beta, g)
$$

By the product rule

$$
D_{t}^{|U|} \prod_{i \in[\hat{T}]} D_{g}^{\left|\hat{T}^{-1}(i)\right|} f^{a}(t, \beta, g)=\sum_{\psi: U \rightarrow[\hat{T}]} \prod_{i \in[\hat{T}]} D_{t}^{\left|\psi^{-1}(i)\right|} D_{g}^{\left|\hat{T}^{-1}(i)\right|} f^{a}(t, \beta, g) .
$$

Set $t=0$. Since $f^{a}(0, \beta, g)=a(\emptyset) g=0$, the contributions from $i$ with $\psi^{-1}(i)=$ $\emptyset$ are zero. So $\psi: U \rightarrow[\hat{T}]$ induces a set partition $\Gamma$ of $U$ and a bijection from 
$\Gamma$ to $[\hat{T}]$. There are $|\hat{T}|$ ! such bijections. This leads to

$$
\left.D_{t}^{|U|} f^{b}\left(1, f^{a}(t, \beta, \cdot), g\right)\right|_{t=0}=\sum_{\Gamma \in \operatorname{Part}[U]} \sum_{\hat{T} \in \mathcal{A}[\Gamma]} c(\Gamma, \hat{T}),
$$

where

$$
c(\Gamma, \hat{T})=\left.\prod_{B \in \Gamma} b(\hat{T}) D_{g}^{\left|\hat{T}^{-1}(B)\right|} D_{t}^{|B|} f^{a}(t, \beta, g)\right|_{t=0} .
$$

Distributive law The next stage is to insert

$$
\left.D_{t}^{|B|} f^{a}(t, \beta, g)\right|_{t=0}=\sum_{H \in \mathcal{A}[B]} a(H) \prod_{j \in B} D_{g}^{\left|H^{-1}(j)\right|} \beta(g)
$$

and use the distributive law. This gives a forest sum

$$
\left.D_{t}^{|U|} f^{b}\left(1, f^{a}(t, \beta, \cdot), g\right)\right|_{t=0}=\sum_{\Gamma \in \operatorname{Part}[U]} \sum_{\hat{T} \in \mathcal{A}[\Gamma]} \sum_{F} c(\Gamma, \hat{T}, F),
$$

where

$$
c(\Gamma, \hat{T}, F)=\prod_{B \in \Gamma} b(\hat{T}) a(F(B)) D_{g}^{\left|\hat{T}^{-1}(B)\right|} \prod_{j \in B} D_{g}^{\left|F(B)^{-1}(j)\right|} \beta(g) .
$$

Product rule The product rule for differentiation produces

$$
c(\Gamma, \hat{T}, F)=\prod_{B \in \Gamma} b(\hat{T}) a(F(B)) \sum_{\left.\phi: \hat{T}^{-1}(B)\right) \rightarrow B} \prod_{j \in B} D_{g}^{\left|\phi^{-1}(j)\right|} D_{g}^{\mid F(B)^{-1}(j \mid} \beta(g) .
$$

Distributive law The distributive law gives

$$
\left.D_{t}^{|U|} f^{b}\left(1, f^{a}(t, \beta, \cdot), g\right)\right|_{t=0}=\sum_{\Gamma \in \operatorname{Part}[U]} \sum_{\hat{T} \in \mathcal{A}[\Gamma]} \sum_{F} \sum_{\Phi} c(\Gamma, \hat{T}, F, \Phi),
$$

where

$$
c(\Gamma, \hat{T}, F, \Phi)=\prod_{B \in \Gamma} \prod_{j \in B} b(\hat{T}) a(F(B)) D_{g}^{\left|\Phi(B)^{-1}(j)\right|} D_{g}^{\mid F(B)^{-1}(j \mid)} \beta(g) .
$$

The sum is over set partitions $\Gamma$ of $U$ and over forest functions $F$ that send block $B$ to rooted tree $F(B)$ on vertex set $B$. It is also over rooted trees $\hat{T}$ on vertex set $\Gamma$. Finally, it is over functions $\Phi$ that send each block $B$ in $\Gamma$ to a function $\Phi(B)$ that takes each $\hat{T}$ preimage block $B^{\prime}$ and sends it to a vertex in $B$.

Construction of rooted tree and subforest These data determine a rooted tree on $U$ that is made from the rooted trees $F(B)$ internal to the blocks $B$ and from the rooted tree $\hat{T}$ and the function $\Phi$. If $\hat{T}\left(B^{\prime}\right)=B$, then there is an edge from the root of the rooted tree on $B^{\prime}$ to the $\Phi(B)\left(B^{\prime}\right)$ in $B$.

The corresponding contribution involves the coefficients $b(\hat{T})$ and $a(F(B))$ and a contribution from the rooted tree. At a given vertex $j$ this involves a 
derivative of $\beta(g)$ of an order equal to the total number of edges impinging on this vertex, both from within the block and from the other blocks.

Giving these data is the same as giving the pair $T \in \mathcal{A}[U]$ together with subforest $F$. So the final expression is

$$
\left.D_{t}^{|U|} f^{b}\left(f^{a}(t, \beta, \cdot), g\right)\right|_{t=0}=\sum_{T \in \mathcal{A}[U]} \sum_{F \sqsubseteq T} a^{\times}(F) b(T / F) f_{T}(g) .
$$

This establishes the result. $\|$

The authors 8 give two applications of this result. For both the idea is to consider the coefficients $e(\tau)=1 / \tau$ ! that give the exact solution of an ordinary differential equation $d x / d t=\beta(x)$. In backward error analysis the idea is to take $c$ corresponding to some numerical method and solve $c=a \star e$ for $a$. This produces a modified differential equation that agrees with the numerical method. In the application to modified integrators, start with a numerical method given by $b$ and solve $e=a \star b$ for $a$. This produces a modified numerical method that agrees with the solution of the differential equation.

\section{Appendix: Algebra and calculus in combinatorics}

Here are some basic results from algebra and calculus in forms that are useful for combinatorics. These are stated in the setting of functions of one variable. There are even more illuminating multi-variable results, but they are not needed in the present exposition.

The distributive law A version of the distributive law of algebra is the following. Suppose that $B$ is a set and for each $b \in B$ there is a corresponding set $F_{b}$. Then the product over $b \in B$ of sums indexed by $F_{b}$ is a sum of products:

$$
\prod_{b \in B} \sum_{t \in F_{b}} a_{b}(t)=\sum_{s} \prod_{b \in B} a_{b}(s(b)) .
$$

The sum on the right is over all functions $s: B \rightarrow \bigcup_{b} F_{b}$ such that for each $b$ the value $s(b) \in F_{b}$. The set of all such functions is the product space $\prod_{b} F_{b}$. In the special case when the $F_{b}=F$ are all the same, the sum is over all functions $s: B \rightarrow F$. In this case the set of all such functions is the Cartesian power space $F^{B}$.

The product rule A version of the product rule for differentiation is the following. Let $U$ be a set with $|U|$ elements. Then the $|U|$ order derivative of a product function is given by

$$
D^{|U|} \prod_{b \in B} F_{b}=\sum_{\phi \in B^{U}} \prod_{b \in B} D^{\left|\phi^{-1}(b)\right|} F_{b} .
$$

Here $B^{U}$ consists of all functions $\phi: U \rightarrow B$. Sometimes a function $\phi$ is described by its inverse images $U_{b}=\phi^{-1}(b)$, so one can think of this as a sum over the corresponding maps $b \mapsto U_{b}$. 
The chain rule A version of the chain rule is the following. Let $U$ be a set with $|U|$ elements. Then the $|U|$ order derivative of a composite function is given by

$$
D^{|U|}(F \circ G)=\sum_{\Gamma \in \operatorname{Part}[U]}\left(D^{|\Gamma|} F\right) \circ G \cdot \prod_{B \in \Gamma} D^{|B|} G .
$$

Here Part $[U]$ consists of all set partitions of $U$ into disjoint non-empty subsets with union $U$.

\section{References}

[1] F. Bergeron, G. Labelle, and P. Leroux, Combinatorial Species and Treelike Structures, Cambridge University Press, Cambridge UK, 1998.

[2] P. Flajolet and RSedgewick, Analytic Combinatorics, Cambridge University Press, Cambridge UK, 2009.

[3] S. MacLane and G. Birkhoff, Algebra, Chelsea, New York, 1988.

[4] G. Bogfjellmo and A. Schmeding, The Lie group structure of the Butcher group, Foundations of Computational Mathematics 17 (2017), 127-159.

[5] Ch. Brouder, Runge-Kutta methods and renormalization, European Physics Journal C 12 (2000), 521-534.

[6] J. C. Butcher, An algebraic theory of integration methods, Mathematics of Computation 26 (1972), 79-106.

[7] D. Calaque, K. Ebrahimi-Fard, and D. Manchon, Two interacting Hopf algebras of trees, Advances in Applied Mathematics 47 (2011), 282-308.

[8] P. Chartier, E. Hairer and G. Vilmart, Algebraic structures of B-series, Foundations of Computational Mathematics 10 (2010), 407-427.

[9] A. Connes and D. Kreimer, Hopf algebras, renormalization and noncommutative geometry, Communications in Mathematical Physics 199 (1998), 203-242.

[10] W. G. Faris, Combinatorics and cluster expansions, Probability Surveys 7 (2010), 157-206.

[11] W. G. Faris, Combinatorial species and Feynman diagrams, Sèminaire Lotharingien de Combinatoire 61A (2011), Article B61An.

[12] F. Girelli, T. Krajewski, and P. Martinetti, An algebraic Birkhoff decomposition for the continuous renormalization group, Journal of Mathematical Physics 45 (2004), 4679-4697.

[13] E. Hairer and G. Wanner, Multistep-multistage-multiderivative methods for ordinary differential equations, Computing 11 (1973), 287-303. 
[14] E. Hairer and G. Wanner, On the Butcher group and general multi-value methods, Computing 13 (1974), 1-15.

[15] A. Kerber, Applied Finite Group Actions, 2nd edition, Springer, Berlin, 1999.

[16] A. Lundervold and H. Munthe-Kaas, Hopf algebras of formal diffeomorphisms and numerical integration on manifolds, in Combinatorics and Physics, Contemporary Mathematics 539 (2011), American Mathematical Society. 